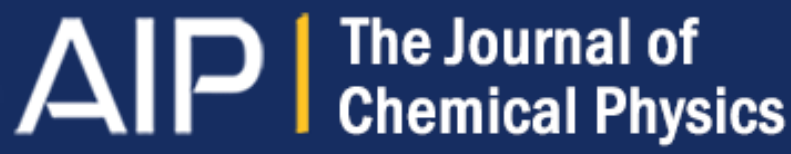

\section{Collective dynamics in molten potassium: An inelastic x-ray scattering study}

A. Monaco, T. Scopigno, P. Benassi, A. Giugni, G. Monaco, M. Nardone, G. Ruocco, and M. Sampoli

Citation: The Journal of Chemical Physics 120, 8089 (2004); doi: 10.1063/1.1689641

View online: http://dx.doi.org/10.1063/1.1689641

View Table of Contents: http://scitation.aip.org/content/aip/journal/jcp/120/17?ver=pdfcov

Published by the AIP Publishing

\section{Articles you may be interested in}

High energy $\mathrm{x}$-ray scattering studies of the local order in liquid $\mathrm{Al}$

J. Chem. Phys. 135, 044502 (2011); 10.1063/1.3609925

Collective dynamics of hydrated $\beta$-lactogloblin by inelastic $x$-ray scattering

J. Chem. Phys. 133, 134501 (2010); 10.1063/1.3484238

An energy dispersive $\mathrm{x}$-ray scattering and molecular dynamics study of liquid dimethyl carbonate

J. Chem. Phys. 131, 244503 (2009); 10.1063/1.3273847

High frequency dynamics in liquid nickel: An inelastic x-ray scattering study

J. Chem. Phys. 128, 234502 (2008); 10.1063/1.2925256

Real-time studies of gallium adsorption and desorption kinetics on sapphire (0001) by grazing incidence smallangle $\mathrm{x}$-ray scattering and $\mathrm{x}$-ray fluorescence

J. Appl. Phys. 103, 103538 (2008); 10.1063/1.2936969

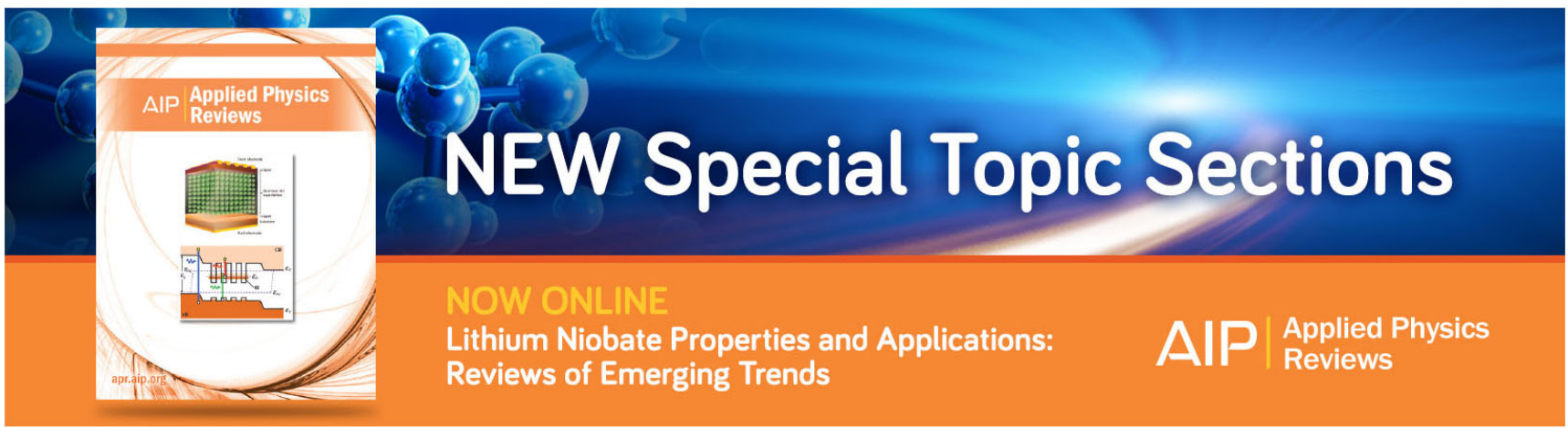




\title{
Collective dynamics in molten potassium: An inelastic x-ray scattering study
}

\author{
A. Monaco \\ Dipartimento di Fisica and INFM, Universitá di L'Aquila, I-67010, L'Aquila, Italy \\ T. Scopigno \\ Dipartimento di Fisica and INFM, Universitá di Roma “La Sapienza," I-00185, Roma, Italy \\ P. Benassi and A. Giugni \\ Dipartimento di Fisica and INFM, Universitá di L'Aquila, I-67010, L'Aquila, Italy \\ G. Monaco \\ European Synchrotron Radiation Facility, B.P. 220 F-38043 Grenoble, Cedex France \\ M. Nardone \\ Dipartimento di Fisica and INFM, Universitá di L'Aquila, I-67010, L'Aquila, Italy \\ G. Ruocco \\ Dipartimento di Fisica and INFM, Universitá di Roma “La Sapienza," I-00185, Roma, Italy \\ M. Sampoli \\ Dipartimento di Energetica and INFM, Universitá di Firenze, I-50019, Firenze, Italy
}

(Received 11 December 2003; accepted 2 February 2004)

\begin{abstract}
The high-frequency collective dynamics of molten potassium has been investigated by inelastic $\mathrm{x}$-ray scattering, disclosing an energy/momentum transfer region unreachable by previous inelastic neutron scattering (INS) experiments. We find that a two-step relaxation scenario, similar to that found in other liquid metals, applies to liquid potassium. In particular, we show how the sound velocity determined by INS experiments, exceeding the hydrodynamic value by $\approx 30 \%$, is the higher limit of a speedup, located in the momentum region $1<Q<3 \mathrm{~nm}^{-1}$, which marks the departure from the isothermal value. We point out how this phenomenology is the consequence of a microscopic relaxation process that, in turn, can be traced back to the presence of "instantaneous" disorder, rather than to the crossover from a liquid to solidlike response. (C) 2004 American Institute of Physics. [DOI: 10.1063/1.1689641]
\end{abstract}

\section{INTRODUCTION}

Alkali metals have been known for a long time to exhibit remarkably pronounced inelastic features (Brillouin peaks) in their density fluctuations frequency spectra, even for wave vectors $(Q)$ well beyond the region of validity of simple hydrodynamics. For this reason, in the last 30 years, the microscopic dynamics of such systems has been widely investigated both experimentally, by means of inelastic neutron scattering (INS), ${ }^{1-8}$ and numerically ${ }^{9-16}$ with the aim of clarifying in detail the mechanisms underlying the atomic motions at a microscopic level.

More recently, the possibility of studying experimentally the microscopic dynamics has been significantly increased by the advent of inelastic x-ray scattering (IXS) spectroscopy. ${ }^{17,18}$ Thanks to this technique, new insight into the collective properties of alkali metals has been provided, ${ }^{19-24}$ allowing the drawing of a much clearer picture of the high-frequency dynamics, so that a deep comprehension of the ultimate nature of the high-frequency excitations in these systems is gradually emerging. In particular, thanks to the development of approaches based on the memory function formalism, ${ }^{25,26}$ a framework has been developed which accounts for the relaxation spectra of the den- sity fluctuations in a large wave vector region. ${ }^{27}$ This framework applies also to non alkali metals, ${ }^{28,29}$ hydrogen-bonded liquids, ${ }^{30,31}$ and polymers. ${ }^{32}$ More specifically, it has been established that the decay of the density-density correlation function occurs through different relaxation mechanisms characterized by different time scales. ${ }^{27}$ These mechanisms appear as additive contributions to the second-order memory function $[M(Q, \omega)]$ of the density fluctuation spectrum. Indeed, in addition to the thermal relaxation process, predicted by the hydrodynamic equations of a simple fluid and arising from the coupling between density and temperature fluctuations, one must also allow explicitly for a non-Markovian behavior of the stress tensor correlation function, thus introducing viscous relaxation. The most palpable consequence of these relaxation processes is that the sound propagation is no longer determined by the hydrodynamic sound velocity.

On the basis of spectroscopic experimental evidences, viscous relaxation is thought to proceed throughout two distinct channels which are active on quite different time scales. ${ }^{27}$ A first rapid decay of $M(Q, \omega)$ [on the time scale $\left.\tau_{\mu}(Q)\right]$ is generally described as arising from the interactions of each atom with its "cage" of surrounding neighbors. This is followed by a slower decay process [time scale $\tau_{\alpha}(Q)$ ] with long-lasting tails which originates from slow, 
temperature-dependent structural rearrangement. Eventually, in those systems capable of undercooling, this rearrangement undergo a structural arrest, thus giving rise to the glass transition phenomenon. Obviously, in order to fully understand the characteristics of these processes, which are in principle common to many liquid systems, thorough investigations in an extended $Q-E$ region are required. Indeed, in the recent past, massive investigations by means of INS, devoted to liquid metals, have produced significant advances in the comprehension of the collective properties of liquids, in particular as far as single-particle properties are concerned. Nevertheless, with the aim of studying collective properties, these approaches have often suffered from limitations, intrinsic to INS, which arise mainly from the presence of incoherent scattering and from kinematic constraints restricting the accessible $Q-E$ region. Very recently, accurate INS experiments have been performed ${ }^{7,8}$ on liquid potassium in which the inelastic components of the spectra have been analyzed either within a memory function framework ${ }^{7}$ or by empirical fitting functions. ${ }^{8}$ In both these studies, values of sound speed exceeding the hydrodynamic one have been reported, and this has been interpreted as a reminiscence of crystallike sound propagation. This interpretation is substantially different from the results reported in other liquid metals $(\mathrm{Li}, \mathrm{Na}$, $\mathrm{Al}, \mathrm{Ga}$ ) (Refs. 22, 24, 28, and 29), and thus deserves a deeper investigation.

\section{EXPERIMENT}

In this work, we present an IXS study of the dynamic structure factor $S(Q, \omega)$ in liquid potassium. Thanks to (i) the purely coherent nature of the IXS cross section in monatomic systems, which gives direct access to the collective dynamic structure factor, and (ii) the lack of kinematic limitations, we have been able to derive convincing information on the ultimate nature of the atomic motion in this system. In particular, we show that, as reported in several other simple liquids ${ }^{27}$ for $Q$ values ranging from just below the diffraction peak maximum $\left(Q_{M} \approx 16 \mathrm{~nm}^{-1}\right)$ all the way down to the hydrodynamic region $\left(Q \approx 1 \mathrm{~nm}^{-1}\right)$, the collective dynamics proceeds basically through two distinct viscous relaxation processes. Within this framework, the observed speed up of the sound velocity, reported by the INS measurements, ${ }^{7,8}$ can be interpreted as due to a relaxation mechanism. In particular, thanks to the accessibility of the low- $Q$ and high- $E$ regions, we show that the process mainly responsible for this increase in the sound velocity is not related to a crossover from a liquidlike to solidlike response. Indeed, although the system is above the melting point, the diffusive motion is frozen on the time scale of the density fluctuation even at the lower investigated $Q$ values [i.e., in terms of the Brillouin peak frequency $\omega_{B}$, one finds $\omega_{B}(Q) \tau_{\alpha}(Q) \gg 1$ over the whole investigated $Q$ range]. On the contrary, the characteristics of the sound propagation are controlled by the faster relaxation induced by the peculiarity of the atomic vibrations associated with the instantaneous positional disorder. ${ }^{16,33}$

The experiment has been carried out at the ID16 beamline of the ESRF in fixed-exchanged wave vector configurations. A typical energy scan $(-50<E<50 \mathrm{meV})$ took about $300 \mathrm{~min}$ and was repeated several times in order to achieve a

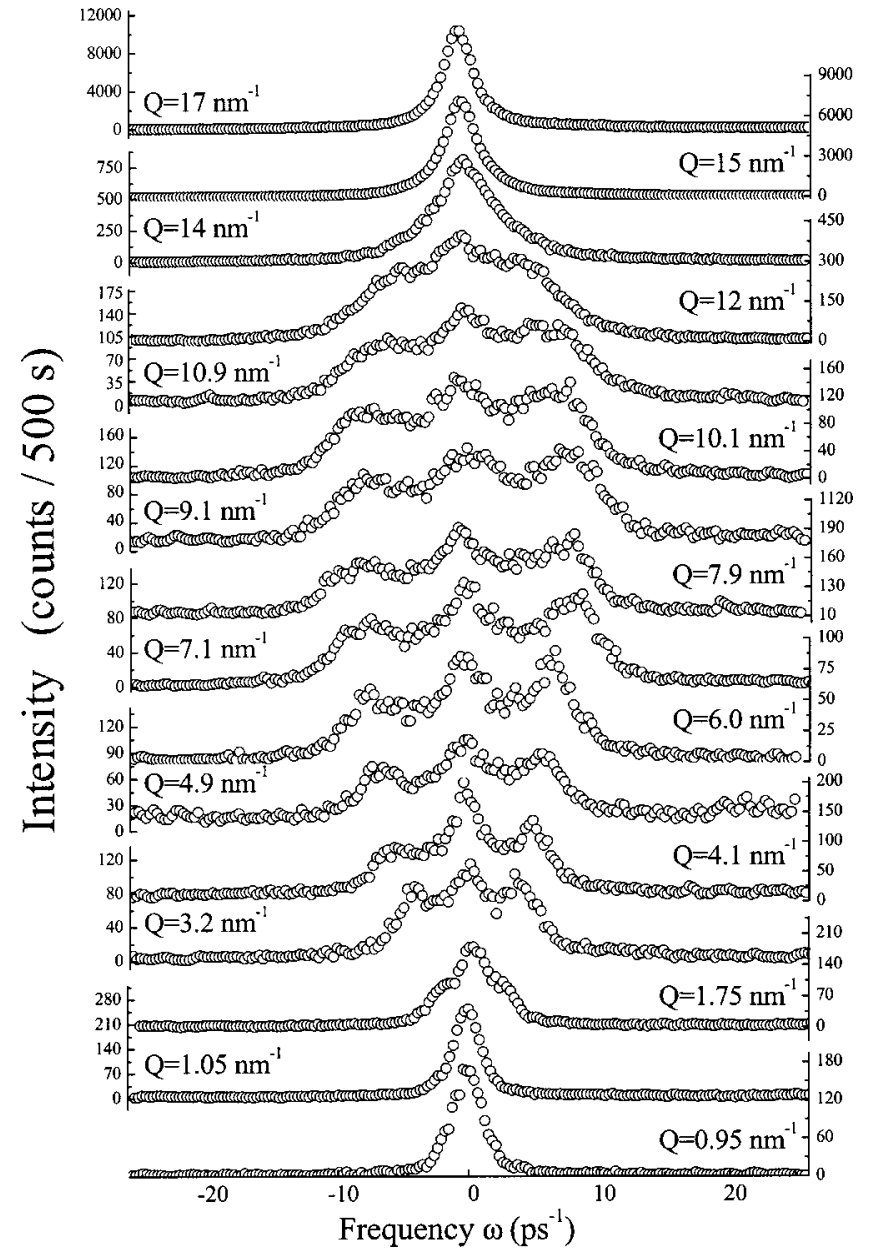

FIG. 1. IXS spectra of liquid potassium $(T=343 \mathrm{~K})$ at the indicated $Q$ values (circles).

total integration time of about $300 \mathrm{~s}$ per point. A fiveanalyzer bench allowed us to collect simultaneously spectra at five different values of the exchanged wave vector $Q$ for each single scan. The sample thickness $(\approx 1 \mathrm{~mm})$ was chosen in order to match the absorption length at the incident energy of $21748 \mathrm{eV}$, corresponding to the (11 11 11) reflection from the silicon analyzers. The energy resolution $\delta E$ in the used configuration was $\approx 1.5 \mathrm{meV}$ full width at half maximum (FWHM). The sample was kept under vacuum at a temperature of $343 \pm 1 \mathrm{~K}$ without the need of any further container windows on either the beam incidence and viewing sides.

In Fig. 1 we report the measured IXS intensity for each investigated (fixed) $Q$ value where the presence of an acoustic propagating mode clearly appears directly from the raw data. Also visible from the data is the presence of a maximum of the dispersion relation (at $Q \approx 10 \mathrm{~nm}^{-1}$ ) and the de Gennes narrowing at $Q$ values close to $Q_{m}$, the position of the main peak of the static structure factor $S(Q)\left(Q_{m}\right.$ $\simeq 16 \mathrm{~nm}^{-1}$ ).

\section{DATA ANALYSIS}

In order to extract quantitative information on these excitations, we performed a data analysis according to the generalized hydrodynamic, ${ }^{25,26}$ already tested against experi- 
mental data available on similar systems. ${ }^{27}$ In this framework, the classical dynamic structure factor can be expressed in terms of its second-order memory function $M(Q, t)$, so that

$$
S(Q, \omega)=\frac{S(Q) \pi^{-1} \omega_{0}^{2}(Q) \tilde{M}^{\prime}(Q, \omega)}{\left[\omega^{2}-\omega_{0}^{2}(Q)+\omega \tilde{M}^{\prime \prime}(Q, \omega)\right]^{2}+\left[\omega \tilde{M}^{\prime}(Q, \omega)\right]^{2}},
$$

where we have introduced the real $\left[M^{\prime}(Q, w)\right]$ and imaginary $\left[M^{\prime \prime}(Q, w)\right]$ parts of the Fourier transform of $M(Q, t)$ and the frequency $\omega_{0}^{2}(Q)=K_{B} T Q^{2} / m S(Q)$, which is related to the generalized isothermal sound speed $c_{t}(Q)$ $=\omega_{0}(Q) / Q$. This latter quantity can be calculated from the liquid structure once $S(Q)$ is known ( $m$ is the atomic mass and $K_{B}$ the Boltzmann constant). In order to fit the data we have accounted for the detailed balance and for the convolution with the instrumental resolution function $R(\omega)$ so that the quantity $I_{M}(Q, \omega)$,

$$
I_{M}(Q, \omega)=\int \frac{\hbar \omega^{\prime} / K T}{1-e^{-\hbar \omega^{\prime} / K T}} S\left(Q, \omega^{\prime}\right) R\left(\omega-\omega^{\prime}\right) d \omega^{\prime},
$$

is compared with the measured scattered intensity $I(Q, \omega)$.

Taking advantage of the results obtained from several other liquid metals, we utilized a memory function characterized by two relevant time scales associated with two processes of viscous origin, besides the usual decay term accounting for thermal relaxation process. This scheme is able to account for the whole $S(Q, \omega)$ features-i.e., for both the quasielastic peak and Brillouin component. To represent the two viscous terms we adopted two simple exponential decays (corresponding to Debye relaxations) to account for the structural $(\alpha)$ relaxation and for the faster, microscopic $(\mu)$ contribution. The thermal relaxation has been described within the usual hydrodinamics result, which also predicts a Debye shape. Consequently, the total memory function reads

$$
\begin{aligned}
M(Q, t)= & (\gamma-1) \omega_{0}^{2}(Q) e^{-D_{T} Q^{2} t}+\Delta_{\alpha}^{2}(Q) e^{-t / \tau_{\alpha}(Q)} \\
& +\Delta_{\mu}^{2}(Q) e^{-t / \tau_{\mu}(Q)} .
\end{aligned}
$$

The value of $\omega_{0}(Q)$ has been calculated using the $S(Q)$ data reported in Ref. 34, while the specific heat ratio $\gamma$ and the thermal diffusion coefficient $D_{T}$ are derived from literature data $^{34}$ neglecting their $Q$ dependence. This latter assumption is substantiated by the observation that in a very similar system-namely, molten lithium- $\gamma(Q)$ (and therefore the magnitude of the thermal relaxation) varies less then $10 \%$ up to $Q_{m}$ (Ref. 12). Although in the large- $Q$ limit $\gamma(Q)$ is expected to approach the limiting value of $5 / 3$, appropriate for a noninteracting monatomic system, any recourse to generalized-hydrodynamics arguments becomes increasingly doubtful for wave vectors distinctly larger than $Q_{m}$.

All these parameters are kept fixed in the fitting procedure, while free fitting parameters are the structural and the microscopic relaxation times $\tau_{\alpha}$ and $\tau_{\mu}$ and the respective relaxation strengths $\Delta_{\alpha}(Q)$ and $\Delta_{\mu}(Q)$. Prior to the fitting, the experimental data have been put in absolute scale by using the first moment sum rule:

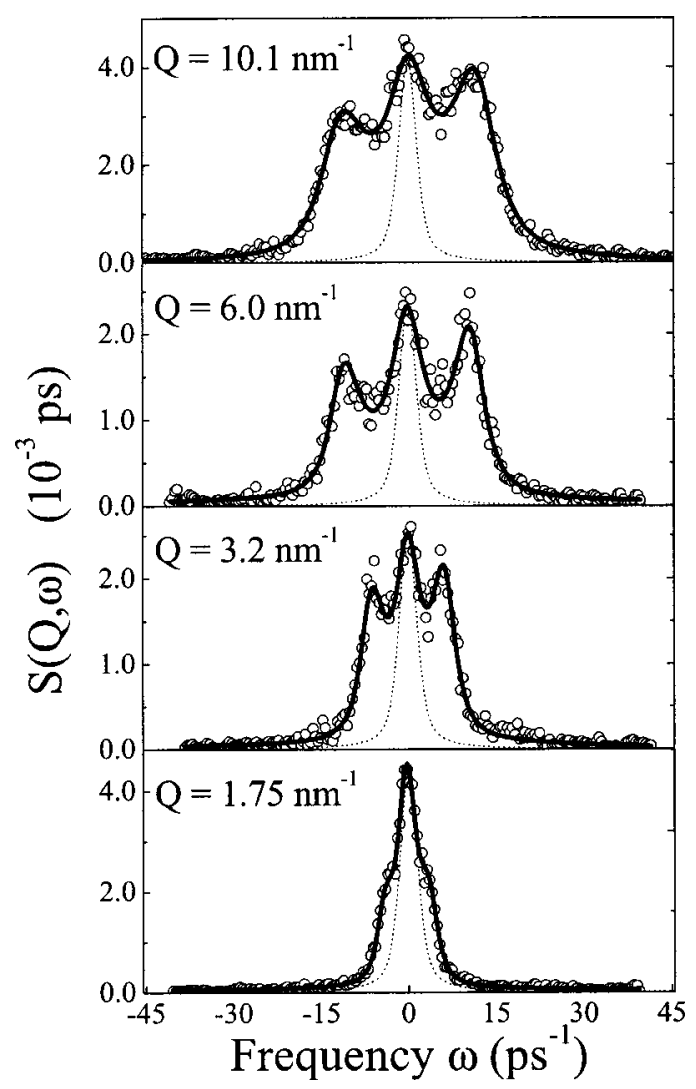

FIG. 2. Experimental $S(Q, \omega)$ (open circles) for selected $Q$ values plotted together with the fitting function (solid line) described in the text. The instrument resolution function (full width at half hight $\delta E \approx 1.5 \mathrm{meV}$ ) is also shown (dotted line).

$$
\begin{aligned}
& \Omega_{\tilde{S}}^{(0)}=\int \widetilde{S}\left(Q, \omega^{\prime}\right) d \omega^{\prime}=\widetilde{S}(Q), \\
& \Omega_{\tilde{S}}^{(1)}=\int \widetilde{S}\left(Q, \omega^{\prime}\right) \omega^{\prime} d \omega^{\prime}=\frac{\hbar Q^{2}}{2 m} .
\end{aligned}
$$

The tilde indicates the dynamical structure factor corrected by the detailed balance prefactor. In terms of the first spectral moments of the measured intensity (subscript $I$ ) and of the resolution function (subscript $R$ ) one can deduce that

$$
\widetilde{S}(Q)=\frac{\hbar Q^{2}}{2 m}\left(\Omega_{I}^{(1)} / \Omega_{I}^{(0)}-\Omega_{R}^{(1)} / \Omega_{R}^{(0)}\right)^{-1}
$$

Consequently, the normalized spectrum reads

$$
I_{N}(Q, \omega)=\widetilde{S}(Q) \frac{I(Q, \omega)}{\int I(Q, \omega) d \omega} .
$$

A comparison between the best fitting line shape and the experimental spectra is reported in Fig. 2 for selected $Q$ values.

It is interesting to test the applicability of the simple viscoelastic model (single relaxation) to the experimental data. As already noticed in several other systems, this oversimplified approximation does not account for the line shape details at the level of accuracy reached by IXS (see Fig. 3).

One of the most relevant pieces of information that can be obtained from an inelastic scattering experiment is the generalized sound velocity, defined as $c_{l}(Q)=\omega_{l}(Q) / Q$ 


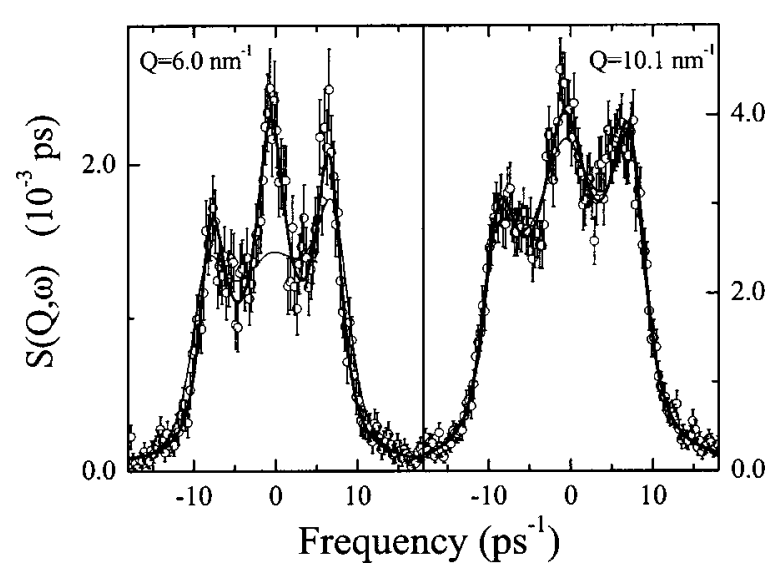

FIG. 3. Detail of IXS spectra of liquid potassium at $Q=6.0$ and $Q$ $=10.1 \mathrm{~nm}^{-1}$. Open circles: experimental data. Thicker line: fit obtained by a model with two viscous relaxation channels. Thinner line: fit results according to the usual viscoelastic model.

(Refs. 25 and 26). The quantity $\omega_{l}(Q)$ corresponds to the position of the maximum of the longitudinal current correlation spectrum given by $J_{L}(Q, \omega)=\left(\omega^{2} / Q^{2}\right) S(Q, \omega)$, which we have calculated using the classical model $S(Q, \omega)$ obtained from our best-fit procedure. In Fig. 4(a) the maxima of the current correlation spectra (solid dots) are compared with
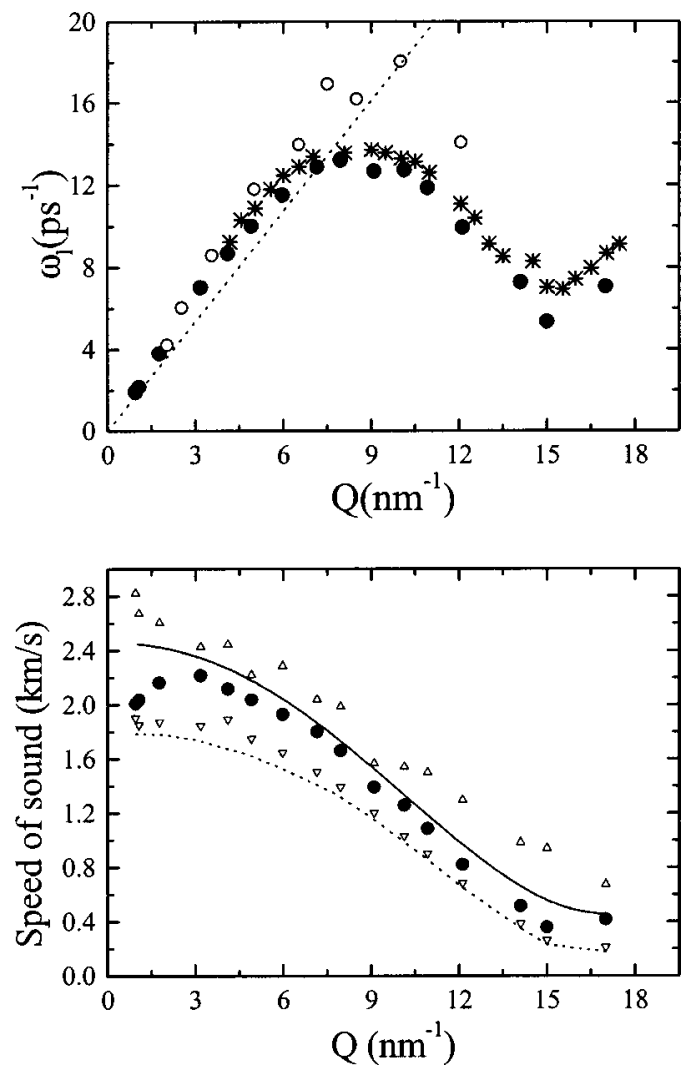

FIG. 4. (a) Maxima of the current correlation spectra $\Omega(Q)$ obtained from the spectra (solid dots) compared with the result of recent INS studies [stars (Ref. 7) and open circles (Ref. 8)]. The dotted line is the hydrodynamic isothermal limit. (b) Sound velocities deduced from the present study: apparent $(\bullet), c_{t}(Q)$ (dotted line) and $c_{\infty}(Q)$ (solid line) deduced by structural data. Also reported are the infinite frequency sound velocity $c_{\infty}(Q)(\triangle)$ and the unrelaxed sound velocity of the structural relaxation process $c_{\infty \alpha}(Q)(\nabla)$ derived from the fit. the result of recent INS studies (stars and open circles). ${ }^{7,8}$ As can be noticed, the IXS data significantly extend at low $Q$ the accessible kinematic region, allowing us to identify the transition from the hydrodynamic region to the high-frequency, mesoscopic regime.

It is worth emphasizing that the quantity which is related to the sound velocity is the maximum of the longitudinal current correlation function. This is, for instance, the quantity which continuously evolves, at increasing wave vectors, towards the single-particle regime, when distinct Brillouin modes are no longer visible in the dynamic structure factor. Moreover, in the framework of generalized hydrodynamics, it is the maximum of $J_{L}(Q, \omega)$ which undergoes a transition between the well-defined quantities $\omega_{0}(Q)$ and $\omega_{\infty}(Q)$ describing the relaxed and unrelaxed mechanical regimes.

In Fig. 4(b) we report the apparent sound velocity (solid dots). In the low- $Q$ region, the measured apparent sound velocity has a maximum $\left[c_{l}\left(Q_{\max }\right) \approx 2240 \mathrm{~m} / \mathrm{s}\right.$ at $Q_{\max }$ $\approx 3 \mathrm{~nm}^{-1}$ ] where it clearly exceeds the isothermal value $(\approx 1790 \mathrm{~m} / \mathrm{s})$ deduced from ultrasonic measurements, ${ }^{34}$ a behavior (the so-called positive dispersion of the sound velocity) that is common with many other simple fluids. It is worth recalling here that, as already pointed out in several IXS studies (see, for example, Ref. 27), in liquid metals, owing to the very high thermal conductivity, in the $Q$ region probed here truly diffusive entropy mode are no longer possible. In other words, the linewidth $\left(D_{T} Q^{2}\right)$ of the thermal diffusion mode exceeds the Brillouin frequency. As a consequence, the positive dispersion of the sound velocity consists of a speedup of the isothermal rather than the adiabatic value, as happens, instead, in usual liquids. In the same figure we also report (i) the generalized ( $Q$-dependent) isothermal sound velocity $\omega_{0}(Q) / Q$ (dotted line), calculated using literature value for $S(Q)$ (Ref. 34), which constitutes the low-frequency limit of the sound speed, and (ii) the infinitefrequency sound velocity $c_{\infty}(Q)$ (solid line), related to the fourth moment sum rule of $S(Q, \omega)$ (Ref. 35) and numerically estimated using the Price-Singwi-Tosi pseudopotential $^{36}$ and a pair distribution evaluated by molecular dynamic simulation, according to the expression ${ }^{25,26}$

$c_{\infty}(Q)=\sqrt{\frac{3 K_{B} T}{m}+\frac{\rho}{m Q^{2}} \int \frac{\partial^{2} V(r)}{\partial z^{2}}\left(1-e^{-i Q z}\right) g(r) d^{3} r}$.

The up triangles are the values of $c_{\infty}(Q)$ deduced from the fit as $c_{\infty}(Q)=\sqrt{\omega_{0}(Q)^{2}+\Delta_{\alpha}(Q)^{2}+\Delta_{\mu}(Q)^{2}} / Q$. The down triangles are the limiting (high-frequency) velocity associated solely with the structural relaxation process: $c_{\infty \alpha}(Q)=\sqrt{\omega_{0}(Q)^{2}+\Delta_{\alpha}(Q)^{2}} / Q-i . e$, , the velocity which is reached when the structural relaxation process is fully unrelaxed $\left[\omega_{B}(Q) \tau_{\alpha}(Q) \gg 1\right]$ or, in other words, the solidlike response. As can be seen, the apparent sound velocity is always larger than $c_{\infty \alpha}(Q)$, indicating the presence of a second relaxation process capable to drive the sound velocity from $c_{\infty \alpha}(Q)$ to $c_{\infty}(Q)$. From this latter observation and from the comparison between $c_{\infty \alpha}(Q)$ and $c_{\infty}(Q)$, it appears evident how the $\alpha$ process only plays a minor role in the full positive dispersion effect. On the other side, it is the faster, micro- 
scopic process [for which we find $\omega_{B}(Q) \tau_{\mu}(Q) \approx 1$ in the whole examined $Q$ range] that pushes the velocity towards a fully unrelaxed regime $\left[c_{\infty}(Q)\right]$.

It is worth mentioning that, in both Refs. 7 and 8 the positive dispersion effect is fully ascribed to the transition between a liquidlike and a solidlike regime-namely, to the structural relaxation process. This claim stems on the basis of the similarity of the sound velocity value of molten potassium with the value for the crystalline acoustic phonons

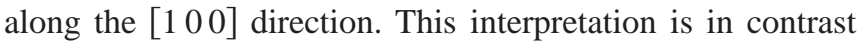
with the results stemming out of the present work as well as with the analogous findings in lithium and sodium. ${ }^{22,24}$ In our opinion, as the positive dispersion is a feature ubiquitous in liquid metals, but not in the crystalline counterpart, one should search for the origin among the specific differences between the two phases: the diffusional motion and the instantaneous disorder. As the first candidate is ruled out by the previous considerations (on the snapshot time scale probed by IXS the liquid appears frozen), it seems natural to associate this effect with the positional disorder of the instantaneous configurations. This experimental fact is substantiated by the evidence of positive dispersion in the microscopic dynamics of simulated glasses. ${ }^{16,33,37}$

Coming back to Fig. 4(b), the slight overestimate of $c_{\infty}(Q)$ values as obtained by the fit (up triangles) with respect to those derived from numerical evaluation has been already recognized as an effect of the exponential shape of $M(Q, t)$ in the $t \rightarrow 0$ limit. $^{27,38}$

A further interesting issue concerns the sound attenuation (Brillouin linewidth): once more the main actor is here the microscopic process, which crosses the resonance condition in the explored IXS $Q$ window $\left[\omega_{B}(Q) \tau_{\mu}(Q)\right.$ always remains of the order of 1]. On the contrary, the "amount of viscosity" $\Delta_{\alpha}^{2} \tau_{\alpha} / Q^{2}$ associated with the structural relaxation does not contribute to the Brillouin width, as a consequence of the solidlike response all over the explored $Q$ range (the Brillouin frequency always exceeds the inverse structural relaxation time).

Within the hydrodynamic framework, the longitudinal viscosity can be generalized accounting for its $Q$ dependence and estimated as the area under the total memory function. In fact, the whole longitudinal viscosity stems from the sum of the structural and microscopic contributions:

$$
\eta_{l}(Q)=\rho\left(\Delta_{\alpha}^{2} \tau_{\alpha}+\Delta_{\mu}^{2} \tau_{\mu}\right) / Q^{2}
$$

We show in Fig. 5 that the low- $Q$ limit obtained by extrapolation of the fitted data is in agreement with the hydrodinamic value $\eta_{l}(Q \rightarrow 0)=1.11 \mathrm{cP}$ (Ref. 34). In the inset of Fig. 5 we plot the single terms of the viscosity associated with the two different processes $\Delta_{\alpha}^{2} \tau_{\alpha}$ and $\Delta_{\mu}^{2} \tau_{\mu}$; we observe that (i) the two contributions are of the same order of magnitude in the investigated $Q$ range and (ii) the microscopic process exhibits a $Q$ dependence which is compatible with a $Q^{2}$ law consistently with the data already reported in other liquids and glasses. ${ }^{27}$

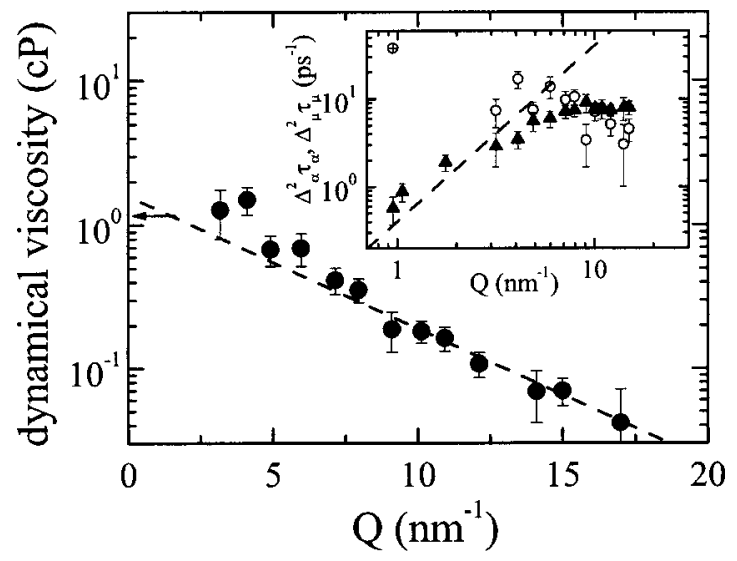

FIG. 5. (a) Values of the longitudinal viscosity $\eta_{l}(Q)$ as determined by the experimental data $(\mathbf{)}$ : dashed line is a guide to the eye. The hydrodynamic value is also reported $(\leftarrow)$. Inset: partial contributions to the $\eta_{l}(Q)$ due to the $\alpha(\bigcirc)$ and $\mu(\mathbf{\Delta})$ processes, respectively. Data for the structural process are reported for $Q \geqslant 3 \mathrm{~nm}^{-1}$ only: as for lower values, the determination of $\tau_{\alpha}$ is unreliable due to finite resolution effects. The dashed line shows the compatibility of the $\mu$ process with a $Q^{2}$ behavior.

\section{CONCLUSION}

We presented an experimental study of the collective high-frequency dynamics in liquid potassium at the melting temperature. Evidence for collective acoustic modes has been found in a $Q$ region extending beyond the hydrodynamic regime up to one-half of the structure factor main peak. A generalized hydrodynamic analysis allows for a quantitative determination of relevant dynamical/ thermodynamical parameters such as the sound velocity, structural relaxation times, and longitudinal viscosity. Although the presented approach is based on the arbitrary choice of the functional form of the memory kernel, it allows one to point out how many relaxation channels are involved in the evolution of the collective dynamics and, more importantly, to determine how they affect the dynamical and transport properties.

The advantages of using a memory function based framework, therefore, are not in a better agreement with the experimental data, but rather in the physical meaning of the involved parameters. This approach, therefore, allows one to gather information on the physical processes underlying the relaxation dynamics and offers the possibility to measure physical quantities and their finite-length-scale generalization. Through this study, for example, we have been able to show how the Brillouin linewidth is not related to the whole longitudinal viscosity (as happens in the usual light scattering regime in ordinary liquids), but rather to the "microscopic" part of the viscosity. In other words, the damping mechanism active in the $\mathrm{THz}$ region does not stem from atomic rearrangements (the viscous flow is frozen out), but is due to the dephasing of the density fluctuations consequent to the non-plane-wave character of the vibrational motion around the quasiequilibrium positions.

Along the same way, the positive dispersion effect can be regarded as a disordered-driven relaxation process with the sound velocity inflection occurring at the resonance condition. On the basis of the reported findings, therefore, the 
interpretation of the positive dispersion in terms of a crystal reminiscent behavior, claimed in Refs. 7 and 8, can be questioned. The viscous to elastic transition, indeed, well observed in the present study and in the whole alkali-metal series, occurs at frequencies below the IXS window and is quantitatively negligible compared to the total soundvelocity increase observed. In this respect, it is worth emphasizing how, in order to gain a quantitatively accurate determination of the sound velocity, one has to look at the maxima of the whole coherent current spectra, rather than arbitrarily distinguishing between a solely inelastic and a quasielastic contribution. ${ }^{25,26}$

In conclusion, this study substantiates previous findings for the collective dynamics in other liquid metals such as $\mathrm{Li}$, $\mathrm{Na}, \mathrm{Al}$, and $\mathrm{Ga}$ (Refs. 22, 24, 28, and 29). This is an important indication of how, despite quantitative differences, the high-frequency dynamics in simple fluids exhibit universal features which go beyond system-dependent details. Since on the observed time scale the structure of the liquid is frozen $\left[\omega_{B}(Q) \tau_{\alpha}(Q) \gg 1\right]$, one can think of the high-frequency dynamics as that of a system with a well-defined equilibrium position (a "glass"). At the same time, the solidlike response alone does not account for the reported spectral features, as the details of the dynamics (sound velocity, microscopic relaxation times, viscosity) are mainly determined by the second, fast, relaxation process which is associated with the vibrations of the instantaneous disordered structure, in agreement with numerical works performed on similar systems. ${ }^{16,33}$

\section{ACKNOWLEDGMENTS}

Valuable help from O. Consorte and the staff of the mechanical workshop of the University of L'Aquila (I) is kindly acknowledged. We also thank L. E. Bove for fruitful discussions.

${ }^{1}$ J. R. D. Copley and M. Rowe, Phys. Rev. A 9, 1656 (1974).

${ }^{2}$ C. Morkel and W. Gläser, Phys. Rev. A 33, 3383 (1986).

${ }^{3}$ T. Bodensteiner, C. Morkel, W. Gläser, and B. Dorner, Phys. Rev. A 45, 5709 (1992).

${ }^{4}$ P. Verkerk, P. H. K. De-Jong, M. Arai, S. M. Bennington, W. S. Howells, and A. D. Taylor, Physica B 180\&181, 834 (1992).

${ }^{5}$ A. G. Novikov, V. V. Savostin, A. L. Shimkevich, and R. M. Yulmetyev, Physica B 228, 312 (1996).

${ }^{6}$ R. Yulmetyev, A. Mokshin, P. Hänggi, and V. Yu Shurygin, Phys. Rev. E 64, 057101 (2001)

${ }^{7}$ C. Cabrillo, F. J. Bermejo, M. Alvarez, P. Verkerk, A. Maira-Vidal, S. M.
Bennington, and D. Martin, Phys. Rev. Lett. 89, 075508 (2002).

${ }^{8}$ L. Bove, B. Dorner, C. Petrillo, F. Sacchetti, and J. Suck, Phys. Rev. B 68, 024208 (2003).

${ }^{9}$ A. Rahman, Phys. Rev. Lett. 32, 52 (1974).

${ }^{10}$ U. Balucani, A. Torcini, and R. Vallauri, Phys. Rev. A 46, 2159 (1992).

${ }^{11}$ U. Balucani, A. Torcini, and R. Vallauri, Phys. Rev. B 47, 3011 (1993).

${ }^{12}$ M. Canales, L. E. Gonzalez, and J. A. Padró, Phys. Rev. E 50, 3656 (1994).

${ }^{13}$ S. Kambayashi and G. Kahl, Phys. Rev. A 46, 3255 (1992).

${ }^{14}$ F. Shimojo, K. Hoshino, and M. Watabe, J. Phys. Soc. Jpn. 63, 141 (1994).

${ }^{15}$ M. Foley, E. Smargiassi, and P. Madden, J. Phys. C 6, 5231 (1994).

${ }^{16}$ T. Scopigno, G. Ruocco, F. Sette, and G. Viliani, Phys. Rev. E 66, 031205 (2002)

${ }^{17}$ E. Burkel, Inelastic Scattering of X-rays with Very High Energy Resolution (Springer-Verlag, Berlin, 1991).

${ }^{18}$ C. Masciovecchio, U. Bergman, M. Krisch, G. Ruocco, F. Sette, and R. Verbeni, Nucl. Instrum. Methods Phys. Res. B 111, 181 (1996).

${ }^{19}$ H. Sinn, F. Sette, U. Bergmann, C. Halcousis, M. Krisch, R. Verbeni, and E. Burkel, Phys. Rev. Lett. 78, 1715 (1997).

${ }^{20}$ W.-C. Pilgrim, S. Hosokawa, H. Saggau, H. Sinn, and E. Burkel, J. NonCryst. Solids 250-252, 96 (1999).

${ }^{21}$ T. Scopigno, U. Balucani, A. Cunsolo, C. Masciovecchio, G. Ruocco, F. Sette, and R. Verbeni, Europhys. Lett. 50, 189 (2000).

${ }^{22}$ T. Scopigno, U. Balucani, G. Ruocco, and F. Sette, Phys. Rev. Lett. 85, 4076 (2000).

${ }^{23}$ T. Scopigno, M. D'Astuto, M. Krisch, G. Ruocco, and F. Sette, Phys. Rev. B 64, 012301 (2001).

${ }^{24}$ T. Scopigno, U. Balucani, G. Ruocco, and F. Sette, Phys. Rev. E 65, 031205 (2002)

${ }^{25}$ J. P. Boon and S. Yip, Molecular Hydrodynamics (McGraw-Hill, New York, 1980)

${ }^{26} \mathrm{U}$. Balucani and M. Zoppi, Dynamics of the Liquid State (Clarendon, Oxford, 1983).

${ }^{27}$ T. Scopigno, U. Balucani, G. Ruocco, and F. Sette, J. Phys. C 12, 8009 (2000).

${ }^{28}$ T. Scopigno, U. Balucani, G. Ruocco, and F. Sette, Phys. Rev. E 63, 011210 (2001).

${ }^{29}$ T. Scopigno, A. Filipponi, M. Krisch, G. Monaco, G. Ruocco, and F. Sette, Phys. Rev. Lett. 89, 255506 (2002).

${ }^{30}$ G. Monaco, A. Cunsolo, G. Ruocco, and F. Sette, Phys. Rev. E 60, 5505 (1999)

${ }^{31}$ R. Angelini, P. Giura, G. Monaco, G. Ruocco, F. Sette, and R. Verbeni, Phys. Rev. Lett. 88, 255503 (2002).

${ }^{32}$ D. Fioretto, M. Mattarelli, C. Masciovecchio, G. Monaco, G. Ruocco, and F. Sette, Phys. Rev. B 65, 224205 (2002).

${ }^{33}$ G. Ruocco, F. Sette, R. Di Leonardo, G. Monaco, M. Sampoli, T. Scopigno, and G. Viliani, Phys. Rev. Lett. 84, 5788 (2000).

${ }^{34} \mathrm{~W}$. Ohse, Handbook of Thermodynamic and Transport Properties of Alkali Metals (Blackwell Scientific, Oxford, 1985).

${ }^{35} \mathrm{An}$ accurate determination of $c_{\infty}(Q)$ from the fourth moment of the dynamic structure factor is actually prevented by the finite instrumental resolution broadening and by background effects which, given the finite frequency range of our data, cannot be easily accounted for.

${ }^{36}$ D. L. Price, K. S. Singwi, and M. P. Tosi, Phys. Rev. B 2, 2983 (1970).

${ }^{37}$ J. Horbach, W. Kob, and K. Binder, Eur. Phys. J. B 19, 531 (2001).

${ }^{38}$ D. Fioretto et al., Phys. Rev. E 59, 4470 (1999). 